\title{
Overexpression of endoglin (CD105) is associated with recurrence in radically resected gastric cancer
}

\author{
YOSHIHISA KOYAMA, HIROKAZU OKAYAMA, KENSUKE KUMAMOTO, \\ KATSUHARU SAITO, IZUMI NAKAMURA, SHINJI OHKI and SEIICHI TAKENOSHITA
}

Department of Organ Regulatory Surgery, Fukushima Medical University School of Medicine, Fukushima 960-1295, Japan

Received March 8, 2010; Accepted May 6, 2010

DOI: 10.3892/etm_00000098

\begin{abstract}
The aim of this study was to examine the expression of endoglin (CD105) in resected gastric cancer and to evaluate the relationship between microvessel density (MVD) and tumor recurrence after surgery. One hundred and thirty-two patients who had undergone curative surgery for primary gastric cancer were immunohistochemically stained for CD105, and MVD was assessed based on the number of CD105-positive vessels. MMP-7 expression was also investigated to determine the relationship with MVD. High MVD was significantly correlated with worse relapsefree survival in all cases and stage I-II cases by log-rank test $(\mathrm{p}=0.0005$ and $\mathrm{p}=0.0154$, respectively). Furthermore, in stage I-II cases, high MVD was the only independent predictor for relapse-free survival by multivariate analysis of the Cox proportional hazard model $[\mathrm{p}=0.028$; hazard ratio $(\mathrm{HR})=4.582$; 95\% confidence interval (CI) 1.184-17.737]. Regarding the specific patterns of recurrence, high MVD was independently related to locoregional recurrence $[\mathrm{p}=0.011$; odds ratio $(O R)=15.208 ; 95 \%$ CI $1.886-122.662]$ and hematogenous recurrence $(\mathrm{p}=0.002 ; \mathrm{OR}=5.718 ; 95 \%$ CI $1.875-17.442)$ by multivariate logistic analysis. However, the expression of MMP-7 or CD105 combined with MVD was associated with relapse-free survival, although these prognostic impacts were not maintained in multivariate analysis. In conclusion, MVD recognized by CD105 may be useful as a predictor for the recurrence of resected gastric cancer and may have a specific association with the development of locoregional and hematogenous recurrence.
\end{abstract}

\section{Introduction}

Gastric cancer is one of the most common malignancies and the major cause of cancer-related death worldwide (1). Surgical resection is the mainstay of treatment for gastric

Correspondence to: Dr Seiichi Takenoshita, Department of Organ Regulatory Surgery, Fukushima Medical University School of Medicine, 1 Hikarigaoka, Fukushima 960-1295, Japan

E-mail: takenoss@fmu.ac.jp

Key words: endoglin, gastric cancer, microvessel density cancer. However, in spite of advances in diagnostic techniques and surgical procedures, the prognosis after resection has remained unsatisfactory due to a high incidence of postoperative recurrence (2). The identification of variables in gastric tumor biology may lead to a more precise assessment of outcome and response to therapy.

Endoglin (CD105) is a $180-\mathrm{kDa}$ homodimeric membrane glycoprotein and the receptor complex of transforming growth factor- $\beta 1$ (3). The CD105 antibody binds preferentially to activated endothelial cells that participate in tumor angiogenesis, and CD105-stained endothelial cells are found on peritumoral and intratumoral vessels. Many studies have examined CD105 expression in a variety of solid tumors, including lung, gastrointestinal, liver, gynecological, prostate and breast (4-8). CD105 is a more specific and sensitive microvessel marker than other traditional panendothelial markers, such as CD31, CD34 and von Willebrand factor. Furthermore, in a variety of solid cancers, CD105 overexpression was consistently associated with poor prognosis and presence of distant metastasis (3). Thus, it has been known that CD105 is an established marker of proliferating tumor blood vessels and a potential predictor of prognosis.

As for gastric cancer, a few studies have demonstrated that overexpression of CD105 in gastric cancer may be associated with shorter overall survival $(9,10)$, although non-curative cases with distant metastasis or peritoneal dissemination were included in these studies. To date, the prognostic value of CD105 in gastric cancer patients after radical surgery has not yet been elucidated. Unfavorable outcomes may be attributed to recurrences that arise from undetectable micrometastases. Without tumor angiogenesis, tumors cannot metastasize to distant organs. Therefore, we hypothesize that the evaluation of neovasculature may be helpful in discriminating the probability of recurrence and poor prognosis in radically resected gastric cancer.

The combination of biomarkers may improve the ability to identify cancer patients at high risk of disease. Therefore, adding an angiogenic factor to the evaluation of CD105 may provide a more clinically useful biomarker than either alone. Matrix metalloproteases (MMPs), which represent a major family of extracellular proteases that target a variety of molecules, are up-regulated in several conditions that accompany angiogenesis. Some family members are implicated in promoting angiogenesis by remodeling the perivascular 
extracellualar matrix $(\mathrm{ECM})$ and liberating angiogenic factors from the ECM (11). Most MMPs are produced by stromal cells, such as fibrobalsts, endothelial cells and inflammatory cells, whereas MMP-7 is primarily expressed by cancer cells (12). These findings may therefore implicate that MMP-7 acts as a specific signal from cancer cells to the stromal cell components necessary for tumor angiogenesis. In fact, the overexpression of MMP-7 is associated with advanced stage and unfavorable prognosis in a variety of tumors (13). Recently, we also reported that MMP-7-expressing tumors have aggressive phenotypes in gastric cancer (14).

In the present study, we investigated the significance of vessels recognized by CD105 as endothelial markers by immunohistochemical staining in 132 cases of curatively resected gastric cancer and analyzed the relationship between microvessel density (MVD) by CD105 and clinical outcomes, including relapse-free survival and the recurrence pattern after surgery. In addition, we used previously acquired data on MMP-7 to evaluate the expression of MMP-7 as an angiogenic factor in relation to the prognostic implication and CD105 expression.

\section{Materials and methods}

Clinical materials. Primary gastric adenocarcinoma specimens were obtained from 132 patients who underwent curative resection at Fukushima Medical University between January 1991 and December 2004. Written informed consent was obtained from all patients before surgery. No patients received chemotherapy or radiotherapy before surgery. Each patient underwent D1 or D2 lymphandectomy. The mean number of examined lymph nodes was 27.71 (15-60) and the mean number of metastatic nodes was 3.59 (0-32). Clinical and pathological status was defined according to the Japanese Classification of Gastric Cancer (15). Histological type was divided into differentiated and undifferentiated type as described previously (14). Routine chemotherapy had been administered to the patients with advanced-stage disease after surgery, but no radiation treatment was carried out in any of the patients included in our study. The endpoint of follow-up was the date of the last contact and the date of death or recurrence through March 2009. The median follow-up time was 1,957 days (range 18-6,407). At the end of our study, 46 (34.8\%) patients had died, 30 (22.7\%) of them directly from gastric cancer after recurrence. The relapse-free 5 -year survival rate was 59.8\%. Of the 132 patients, 33 (25\%) showed recurrence during the postoperative follow-up period. Hematogenous, peritoneal and locoregional recurrences were observed in 22, 12 and 11 cases, respectively. Some patients had overlapping recurrence.

Immunohistochemistry. All specimens were fixed in formalin and embedded in paraffin. Serial sections $(4 \mu \mathrm{m})$ were deparaffinized in xylene and hydrated through a graded series of ethanol. After the sections were rinsed in phosphate-buffered saline (PBS), endogenous peroxidase was blocked with $0.3 \%$ $\mathrm{H}_{2} \mathrm{O}_{2}$ in methanol for $30 \mathrm{~min}$. Antigens were retrieved by autoclaving sections on slides in $0.01 \mathrm{M}(\mathrm{pH}$ 6.0) citrate buffer for $10 \mathrm{~min}$ for MMP-7, or by incubation with Proteinase K for $5 \mathrm{~min}$ for $\mathrm{CD} 105$. After being rinsed in PBS, the sections were incubated with each primary antibody overnight at $4^{\circ} \mathrm{C}$.
The primary antibodies were anti-CD105 (clone SN6h; R\&D Systems, Minneapolis, MN, USA; 1:40) and anti-MMP-7 (clone 141-7B2; Daiichi Fine Chemical, Toyama, Japan; 1:200). A further wash in PBS was followed by treatment with peroxidase-labeled polymer conjugated to goat anti-mouse immunogloblins (Envison ${ }^{+}$kit; Dako, Glostrup, Denmark) as the secondary antibody for $30 \mathrm{~min}$ at room temperature. The staining was visualized with diaminobenzidine (DAB), followed by counterstaining with hematoxylin.

Sections were considered positive for MMP-7 when $>5 \%$ of tumor cells were stained in the cytoplasm or cell membrane. Assessment of the staining was evaluated by two independent pathologists without knowledge of the clinical status of the patients.

Quantification of MVD. The MVD recognized by CD105 was evaluated under light microscopy according to the procedure described by Weidner et al (16). Briefly, after scanning the sections at low magnifications (x40), three tumor areas with the greatest number of distinctly highlighted micovessels ('hot spot') were selected. The number of vessels was counted in the hot spots at high magnifications (x200), and the average counts of the fields were recorded. Each brown-stained endothelial cell or endothelial cell cluster, which was clearly separate from the adjacent micovessels, tumor cells and connective tissue elements was considered a single, countable microvessel.

Statistical analysis. The Student's t-test and one-way ANOVA were used to compare means in groups. We used the Bonferoni test to compare multiple pairs. Cumulative survival was estimated by the Kaplan-Meier method, and differences between survival curves were analyzed by the log-rank test. The influence of each variable on survival was analyzed by the multivariate analysis of Cox proportional hazard model. To identify the independent predictors for each recurrence pattern, the factors found to be significant in univariate analysis were included in subsequent multivariate logistic regression analysis. Differences at $\mathrm{p}<0.05$ were considered significant. All statistical analyses were performed using SPSS 11.0 software (SPSS Inc., Chicago, IL, USA).

\section{Results}

CD105 and MMP-7 expression in gastric cancer. In normal gastric tissues, CD105 staining was hardly observed in any vessels (Fig. 1A). CD105-stained microvessels were frequently observed in microvessels of tumoral tissues (Fig. 1B-D). The mean MVD value as assessed by CD105 was $37.20 \pm 23.72$ (mean $\pm \mathrm{SD}$, median 32.55, range 3.67-94.33). As shown in Table I, higher MVD was significantly correlated with deeper depth of invasion $(\mathrm{p}=0.001)$, presence of lymphatic invasion $(\mathrm{p}=0.022)$, presence of venous invasion $(\mathrm{p}=0.001)$, presence of lymph node metastasis $(\mathrm{p}<0.001)$ and advanced stage $(\mathrm{p}<0.001)$. No correlation was found between MVD and age, gender and histological type.

The positive expression of MMP-7 (Fig. 1F-H), which existed in the cytoplasm and membrane of cancer cells, was found in 95 of the 132 cases (72\%), while MMP-7 expression was rarely detected in normal endothelial cells (Fig. 1E). 

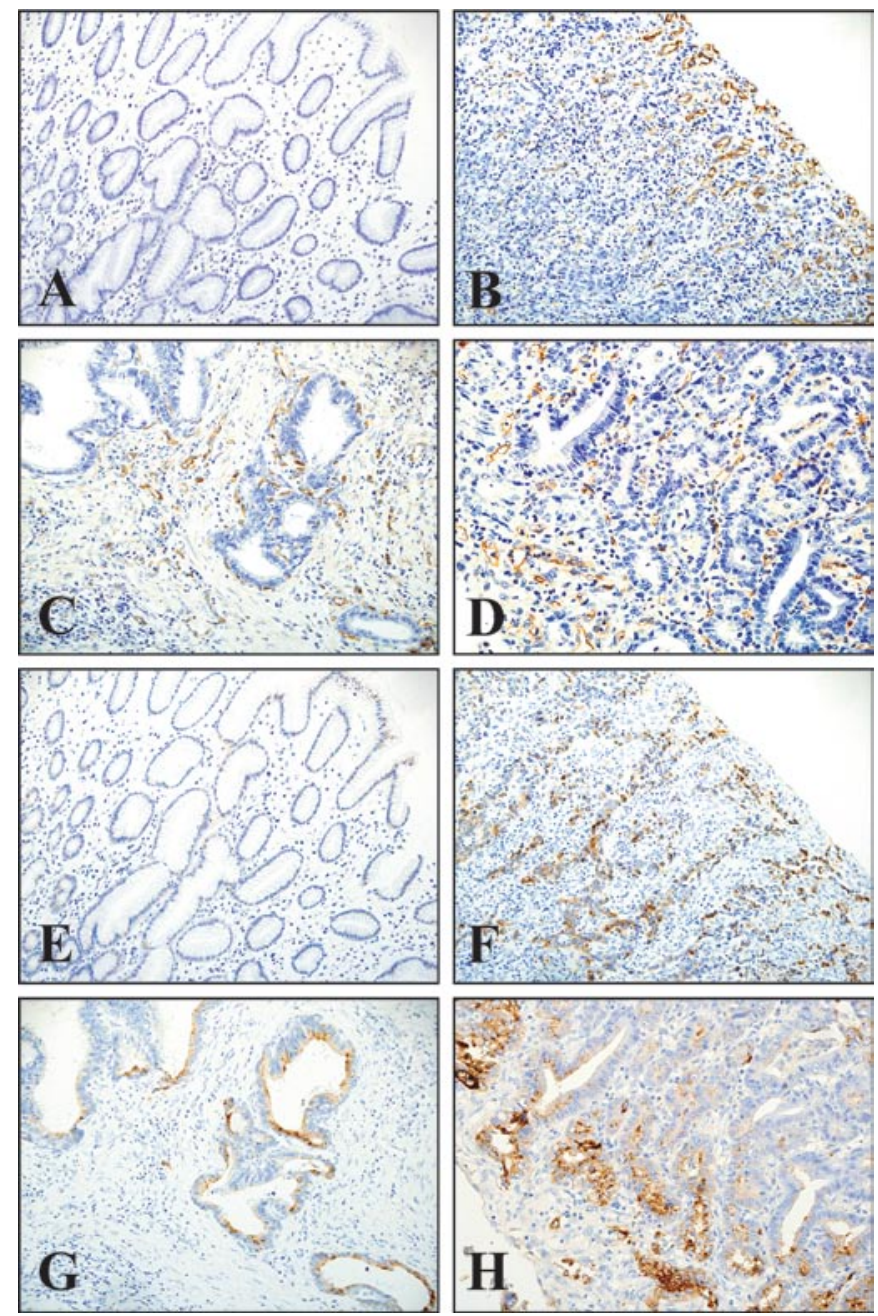

Figure 1. Sequential sections subjected to CD105 staining (A-D) and MMP-7 staining (E-H). (A) Normal gastric mucosa showing no CD105 staining (original magnification $\mathrm{x} 20$ ). ( $\mathrm{B}, \mathrm{C}$ and $\mathrm{D}$ ) In gastric cancer tissues, endothelial cells of small capillary-like vessels were stained with CD105 (x20, x20 and $x 40$ ). (E) MMP-7 expression was rarely detected in normal endothelial cells (x20). (F, G and H) MMP-7 was expressed in the cytoplasm and membrane of cancer cells (x20, x20 and $\times 40)$.

Higher MVD was also associated with positive MMP-7 expression $(\mathrm{p}=0.019)$.

Prognostic significance of MVD by CD105 and MMP-7 expression. The median rate of MVD was 32.55 , and the value of 35 was chosen as the cut-off point. High and low MVD was observed in 58 (43.9\%) and 74 (56.1\%) patients, respectively. Fig. 2A shows the survival curves of the patients according to high or low MVD; the survival rates were evaluated by log-rank test. High-MVD patients were significantly associated with worse relapse-free survival compared to low-MVD patients $(\mathrm{p}=0.0005)$. Similarly, patients with MMP-7 expression also showed significant worse relapse-free survival $(\mathrm{p}=0.0293)$ (Fig. 2B). We further performed the combined analysis of MVD and MMP-7 expression to predict recurrence. As shown in Fig. 2C, high-MVD and positive MMP-7 patients had the worst prognosis, whereas low-MVD and negative MMP-7 patients had the most favorable prognosis $(p=0.0010)$. However, combined analysis of MVD and MMP-7
Table I. MVD by CD105 and clinicopathological factors in patients with gastric cancer.

\begin{tabular}{|c|c|c|c|}
\hline & $\begin{array}{l}\text { Patients } \\
\mathrm{n}(\%)\end{array}$ & $\begin{array}{c}\text { MVD by CD105 } \\
\text { mean }(\mathrm{SD})\end{array}$ & p-value \\
\hline Age & & & 0.251 \\
\hline$>65$ & $70(53)$ & $34.96(24.13)$ & \\
\hline$<65$ & $62(47)$ & $39.73(23.19)$ & \\
\hline Gender & & & 0.837 \\
\hline Male & $89(67)$ & 37.49 (25.16) & \\
\hline Female & $43(33)$ & $36.58(20.71)$ & \\
\hline Histological type & & & 0.779 \\
\hline Differentiated & $63(48)$ & $36.59(26.00)$ & \\
\hline Undifferentiated & $69(52)$ & $37.75(21.61)$ & \\
\hline Depth of invasion & & & 0.005 \\
\hline $\mathrm{T} 1$ & $41(31)$ & $27.44(23.08)$ & \\
\hline $\mathrm{T} 2$ & $54(41)$ & $42.61(23.26)$ & \\
\hline $\mathrm{T} 3$ & $37(28)$ & $40.11(22.33)$ & \\
\hline Lymphatic invasion & & & 0.022 \\
\hline Present & $107(81)$ & $39.47(23.23)$ & \\
\hline Absent & $25(19)$ & $27.48(23.79)$ & \\
\hline Venous invasion & & & 0.001 \\
\hline Present & $94(71)$ & $41.49(24.56)$ & \\
\hline Absent & $38(29)$ & $26.53(17.72)$ & \\
\hline LN metastasis & & & $<0.001$ \\
\hline Positive & $70(53)$ & $46.64(23.46)$ & \\
\hline Negative & $62(47)$ & $26.53(19.18)$ & \\
\hline Stage & & & $<0.001$ \\
\hline I & $60(45)$ & $25.20(18.48)$ & \\
\hline II & $28(21)$ & $49.50(21.76)$ & \\
\hline III & $44(34)$ & $45.73(23.97)$ & \\
\hline MMP-7 expression & & & 0.019 \\
\hline Positive & $95(72)$ & $40.06(24.13)$ & \\
\hline Negative & $37(28)$ & $29.84(21.22)$ & \\
\hline Recurrence & & & 0.003 \\
\hline Yes & $33(25)$ & $47.76(25.15)$ & \\
\hline No & $99(75)$ & $33.68(22.26)$ & \\
\hline
\end{tabular}

was not superior to that of MVD alone in both univariate and multivariate analysis.

To evaluate the potential use of MVD and MMP-7 as a clinical biomarker, we performed stratified analysis by stage of disease (Fig. 3A and B). There was a significant difference between high MVD and relapse-free survival in stage I-II cases $(\mathrm{p}=0.0154)$, but not in stage III cases $(\mathrm{p}=0.4239)$. However, when stratified by stage of disease, MMP-7 expression was not significantly related to relapse-free survival (data not shown).

The prognostic relevance of MVD and MMP-7 was assessed using univariate and subsequent multivariate Cox proportional hazard model adjusted for the established clinical prognostic factors in all cases and stage I-II cases, respectively (Table IIA and B). The multivariate analysis revealed that depth of invasion $[\mathrm{p}<0.001$; hazard ratio $(\mathrm{HR})=2.001$; 95\% confidence interval (CI) 1.396-2.868] and lymph node 
A

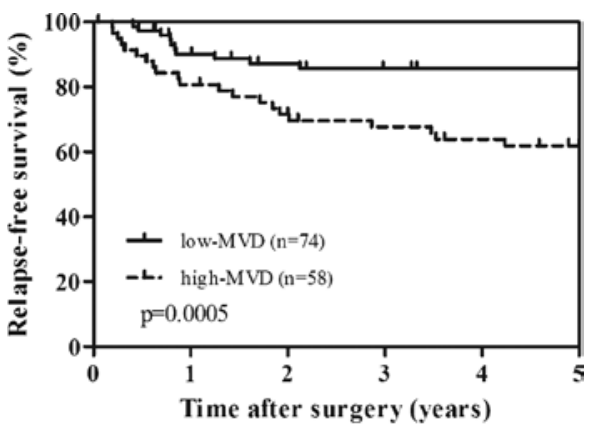

B

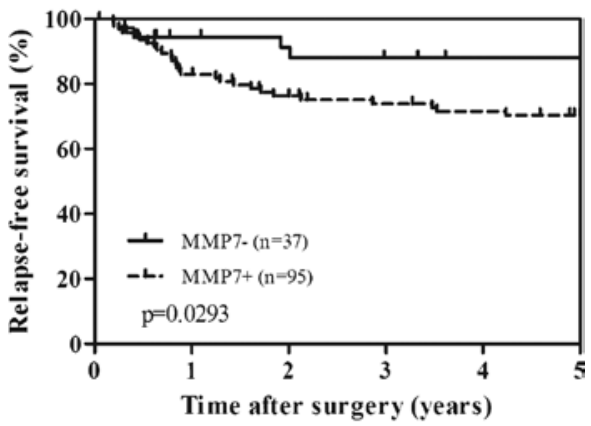

C

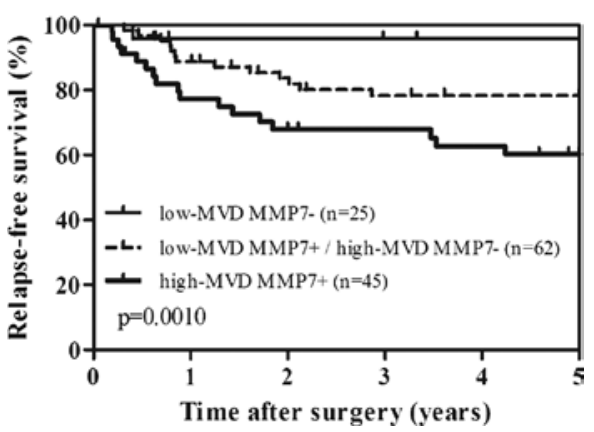

Figure 2. Kaplan-Meier curves of relapse-free survival according to MVD by CD105 (A), MMP-7 expression (B) and combined MVD and MMP-7 expression $(\mathrm{C})$ in patients with resected gastric cancer. The p-values were determined by the log-rank test.

metastasis $(\mathrm{p}<0.001 ; \mathrm{HR}=5.274 ; 95 \%$ CI 1.797-15.479) were independently associated with a relapse-free survival in all cases. When restricting the analysis to stage I-II cases, high MVD was the only independent prognostic factor associated with relapse-free survival $(\mathrm{p}=0.028 ; \mathrm{HR}=4.582 ; 95 \%$ CI 1.184 17.737). However, MMP-7 expression did not predict survival independently.

Relation between recurrence patterns and MVD. The relations between the recurrence patterns and MVD counts are shown in Fig. 4. The mean MVD in patients without recurrence, patients with peritoneal, hematogenous and locoregional recurrence was $33.68 \pm 22.26,35.08 \pm 25.25,50.55 \pm 23.77$ and $58.82 \pm 26.10$, respectively. Higher MVD was significantly correlated with hematogenous and locoregional recurrence $(p<0.05$ and $\mathrm{p}<0.01$, respectively). By contrast, no relationship was found between MMP-7 expression and any specific patterns of recurrence. To determine whether any variable would provide a better estimate of relative risks for the development of metastasis, univariate and subsequent multivariate logistic analysis was applied. Covariants included histological type,
A

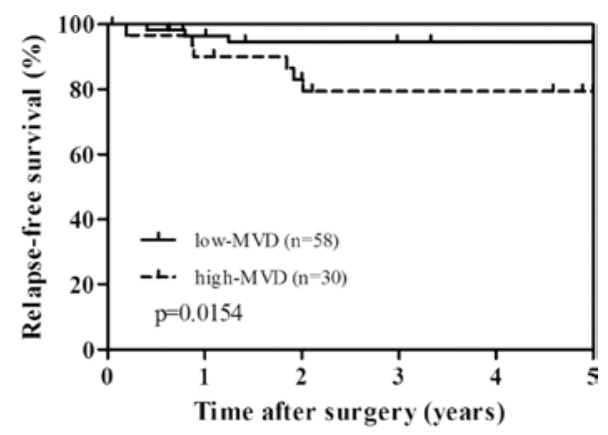

B

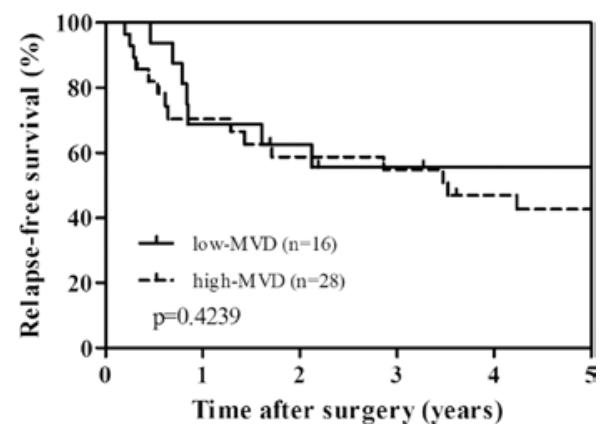

Figure 3. Kaplan-Meier curves of relapse-free survival according to MVD by CD105, stratified by clinical stage, showing stage I-II (A) and stage III (B). The p-values were determined by the log-rank test.

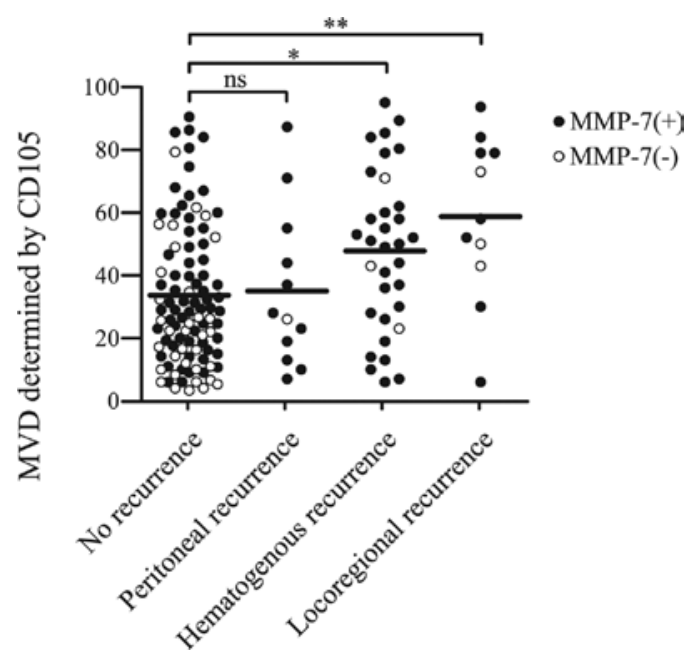

Figure 4. Comparison of MVD determined by CD105 with specific site of recurrence. MMP-7 expression was also determined. MVD was significantly higher in patients with hematogenous recurrence or locoregional recurrence. " $\mathrm{p}<0.05,{ }^{* *} \mathrm{p}<0.01$.

depth of invasion, lymphatic invasion, venous invasion, lymph node metastasis, MVD and MMP-7 expression. As shown in Table III, multivariate analysis identified T3 (serosal invasion) tumor, presence of lymph node metastasis and high-MVD as independent predictors of recurrence. With respect to each recurrence pattern, depth of invasion was associated with both hematogenous and peritoneal recurrence. On the other hand, high MVD was associated with both hematogenous and locoregional recurrence. However, histological type, lymphatic or venous invasion and MMP-7 expression were not associated with any specific recurrence pattern in multivariate analysis. 
Table II. Univariate and multivariate Cox regression analysis of relapse-free survival.

A, All cases (stage I-III cases).

\begin{tabular}{|c|c|c|c|c|c|}
\hline \multirow[t]{2}{*}{ Variable } & \multirow[t]{2}{*}{ Comparison/referent } & \multirow{2}{*}{$\frac{\text { Univariate }}{\text { p-value }}$} & \multicolumn{3}{|c|}{ Multivariate } \\
\hline & & & HR & $95 \% \mathrm{CI}$ & p-value \\
\hline MVD by CD105 & High/low & 0.001 & & & NS \\
\hline MMP-7 & Positive/negative & 0.038 & & & NS \\
\hline Age & $>60 /<60$ & 0.076 & & & \\
\hline Gender & Female/male & 0.729 & & & \\
\hline Histological type & Undifferentiated/differentiated & 0.417 & & & \\
\hline Depth of invasion & $\mathrm{T} 3 / \mathrm{T} 1-\mathrm{T} 2$ & $<0.001$ & 2.001 & $1.396-2.868$ & $<0.001$ \\
\hline Lymph node metastasis & Present/absent & $<0.001$ & 5.274 & $1.797-15.479$ & 0.002 \\
\hline
\end{tabular}

B, Stage I-II cases.

\begin{tabular}{|c|c|c|c|c|c|}
\hline \multirow[t]{2}{*}{ Variable } & \multirow[t]{2}{*}{ Comparison/referent } & \multirow{2}{*}{$\frac{\text { Univariate }}{\text { p-value }}$} & \multicolumn{3}{|c|}{ Multivariate } \\
\hline & & & HR & $95 \% \mathrm{CI}$ & $\mathrm{p}$-value \\
\hline MVD by CD105 & High/low & 0.028 & 4.582 & $1.184-17.737$ & 0.028 \\
\hline MMP-7 & Positive/negative & 0.657 & & & \\
\hline Age & $>60 /<60$ & 0.127 & & & \\
\hline Gender & Female/male & 0.831 & & & \\
\hline Histological type & Undifferentiated/differentiated & 0.721 & & & \\
\hline Depth of invasion & $\mathrm{T} 3 / \mathrm{T} 1-\mathrm{T} 2$ & 0.680 & & & \\
\hline Lymph node metastasis & Present/absent & 0.045 & & & NS \\
\hline
\end{tabular}

HR, hazard ratio; CI, confidence interval; NS, not significant.

Table III. Multivariate analysis for each recurrence pattern by logistic regression.

\begin{tabular}{lccc}
\hline & Comparison/referent & OR & 95\% CI \\
\hline Any recurrence & & & \\
$\quad$ Depth of invasion & T3/T1-T2 & 2.325 & $1.418-3.812$ \\
Lymph node metastasis & Present/absent & 4.089 & $1.171-14.285$ \\
MVD by CD105 & High/low & 2.882 & $1.025-8.108$ \\
Locoregional recurrence & & & 0.001 \\
MVD by CD105 & High/low & 15.208 & 0.045 \\
Hematogenous recurrence & & & $1.886-122.662$ \\
Depth of invasion & T3/T1-T2 & 2.274 & $1.365-3.789$ \\
MVD by CD105 & High/low & 5.718 & 0.002 \\
Peritoneal recurrence & & & 0.002 \\
Depth of invasion & T3/T1-T2 & 4.148 & $1.885-9.126$ \\
\hline
\end{tabular}

OR, odds ratio; CI, confidence interval.

\section{Discussion}

Early diagnosis, surgical treatment with systematic lymph node dissection and appropriate chemotherapy have improved the survival of patients with gastric cancer (17). However, even after a curative resection, tumor recurrences are likely to assume a variety of forms in various organs. The prediction of risks for recurrences as well as recurrence patterns after surgery could help the design of better follow-up programmes and appropriate treatment strategies for gastric cancer patients. Recurrences probably arise from the growth of occult micrometastases that have already been established at the time 
of surgery. This may depend on the biological nature of the resected tumor itself.

CD105 is a proliferation-associated and hypoxia-inducible glycoprotein abundantly expressed in angiogenic endothelial cells, and it is essential in angiogenesis. The intensity of staining for CD105 is greater in blood vessel endothelia within neoplastic than within normal tissues, indicating that CD105 is a powerful marker of neovascularization in solid malignancies (3).

Herein, we showed for the first time that MVD by CD105 indicates recurrence in patients with resected gastric cancer. Significant higher MVD was found in tumors with deeper depth of invasion, presence of lymphatic and venous invasion, presence of lymph node metastasis, advanced stage and tumor recurrence. In survival analysis, high-MVD was significantly correlated with worse relapse-free survival by univariate analysis. When tumors were divided into stage I-II and stage III, high MVD was also significantly associated with worse relapse-free survival in stage I-II cases. Furthermore, in stage I-II cases, high MVD was the only independent predictor for relapse-free survival by multivariate analysis of Cox proportional hazard model. Regarding the specific patterns of recurrence, high MVD was independently related to locoregional and hematogenous recurrence by multivariate logistic analysis.

MMP-7 plays a key role, not only in the degradation of extracellular matrix (ECM), but also in the creation and maintenance of a microenvironment that facilitates the growth and angiogenesis of tumors $(12,13)$. MMP-7 accelerates the proliferation of human umbilical vein endothelial cells in vitro (18). Another study demonstrated that MMP-7-induced angiogenesis in a mouse model was inhibited by an MMP-7 specific antisense oligonucleotide (19). Moreover, MMP-7 cleaves the matrix-bound isoform of vascular endothelial growth factor, which is one of the most powerful mediators of angiogenesis (20). These findings suggest that MMP-7 potently promotes angiogenesis. In the present study, we revealed that MMP-7 expression in tumors was significantly related to MVD recognized by CD105, suggesting that MMP-7 may be involved in neoangiogenesis in gastric cancer. Furthermore, patients with MMP-7-positive tumor had shorter relapse-free survival. However, multivariate analysis revealed that MMP-7 expression lost independence of development of recurrence.

In the present study, we also revealed that higher MVD was significantly correlated with lymph node status and lymphatic invasion, suggesting that CD105 may be involved in lymphatic metastasis. Recently, Clasper et al demonstrated that CD105 was up-regulated in tumor lymphatic endothelial cells (LEC) compared to normal LEC, using combined GeneChip microarray and immunohistochemical analyses. The authors also revealed that CD105 was not confined to the blood vasculature, but detected in numerous LYVE-1-positive tumor lymphatics (21). Likewise, Yoshitomi et al reported that CD105 was found in lymphatic endothelial cells of pancreatic cancer tissue identified by staining with the D2-40 antibody (22). These results suggest that CD105 expression in endothelial cells of small capillary-like vessels consist of immature endothelial cells induced, not only by tumor angiogenesis, but also by tumor lymphangiogenesis. Our finding was consistent with the reports that CD105 also exists in lymphatics, suggesting that CD105 may be a potential predictor of lymphatic metastasis as well as hematogenous metastasis.

These data suggest that CD105 is a candidate target molecule for novel antitumor therapy based on the inhibition of tumor neovasculature. In fact, anti-CD105 therapy has been validated experimentally in several animal models $(3,23,24)$. Recently, targeting the angiogenic mediator vascular endothelial growth factor has proven efficacious in several solid malignancies, therefore, targeting the tumor-associated activated endothelial cell directly may also be a successful strategy (25).

In conclusion, MVD recognized by CD105 may be a useful predictor of tumor recurrence and specific site of reccurence after surgical resection, and thereby may help to refine therapeutic decisions in gastric cancer.

\section{References}

1. Jemal A, Siegel R, Ward E, Hao Y, Xu J and Thun MJ: Cancer statistics, 2009. CA Cancer J Clin 59: 225-249, 2009.

2. Moriguchi S, Maehara Y, Korenaga D, Sugimachi K and Nose Y: Risk factors which predict pattern of recurrence after curative surgery for patients with advanced gastric cancer. Surg Oncol 1: 341-346, 1992.

3. Dallas NA, Samuel S, Xia L, Fan F, Gray MJ, Lim SJ and Ellis LM: Endoglin (CD105): a marker of tumor vasculature and potential target for therapy. Clin Cancer Res 14: 1931-1937, 2008.

4. Saad RS, Liu YL, Nathan G, Celebrezze J, Medich D and Silverman JF: Endoglin (CD105) and vascular endothelial growth factor as prognostic markers in colorectal cancer. Mod Pathol 17: 197-203, 2004.

5. Mineo TC, Ambrogi V, Baldi A, Rabitti C, Bollero P, Vincenzi B and Tonini G: Prognostic impact of VEGF, CD31, CD34, and CD105 expression and tumour vessel invasion after radical surgery for IB-IIA non-small cell lung cancer. J Clin Pathol 57: 591-597, 2004

6. Zijlmans HJ, Fleuren GJ, Hazelbag S, Sier CF, Dreef EJ, Kenter GG and Gorter A: Expression of endoglin (CD105) in cervical cancer. Br J Cancer 100: 1617-1626, 2009.

7. El-Gohary YM, Silverman JF, Olson PR, Liu YL, Cohen JK, Miller R and Saad RS: Endoglin (CD105) and vascular endothelial growth factor as prognostic markers in prostatic adenocarcinoma. Am J Clin Pathol 127: 572-579, 2007.

8. Li C, Guo B, Wilson PB, Stewart A, Byrne G, Bundred N and Kumar S: Plasma levels of soluble CD105 correlate with metastasis in patients with breast cancer. Int J Cancer 89: 122-126, 2000.

9. Ding S, Li C, Lin S, Yang Y, Liu D, Han Y, Zhang Y, Li L, Zhou L and Kumar S: Comparative evaluation of microvessel density determined by CD34 or CD105 in benign and malignant gastric lesions. Hum Pathol 37: 861-866, 2006.

10. Nikiteas NI, Tzanakis N, Theodoropoulos G, Atsaves V, Christoni Z, Karakitsos P, Lazaris AC, Papachristodoulou A, Klonaris $\mathrm{C}$ and Gazouli M: Vascular endothelial growth factor and endoglin (CD-105) in gastric cancer. Gastric Cancer 10: 12-17, 2007.

11. Roy R, Zhang B and Moses MA: Making the cut: proteasemediated regulation of angiogenesis. Exp Cell Res 312: 608-622, 2006.

12. Overall $\mathrm{CM}$ and Kleifeld $\mathrm{O}$ : Tumour microenvironment opinion: validating matrix metalloproteinases as drug targets and anti-targets for cancer therapy. Nat Rev Cancer 6: 227-239, 2006.

13. Ii M, Yamamoto H, Adachi Y, Maruyama Y and Shinomura Y: Role of matrix metalloproteinase-7 (matrilysin) in human cancer invasion, apoptosis, growth, and angiogenesis. Exp Biol Med 231: 20-27, 2006.

14. Okayama H, Kumamoto K, Saitou K, Hayase S, Kofunato Y, Sato Y, Miyamoto K, Nakamura I, Ohki S, Sekikawa K and Takenoshita S: CD44v6, MMP-7 and nuclear Cdx2 are significant biomarkers for prediction of lymph node metastasis in primary gastric cancer. Oncol Rep 22: 745-755, 2009.

15. Japanese Gastric Cancer Association: Japanese classification of gastric carcinoma, 2nd English edition. Gastric Cancer 1: 10-24, 1998. 
16. Weidner N, Semple JP, Welch WR and Folkman J: Tumor angiogenesis and metastasis - correlation in invasive breast carcinoma. N Engl J Med 324: 1-8, 1991.

17. Hartgrink HH, Jansen EP, van Grieken NC and van de Velde CJ: Gastric cancer. Lancet 374: 477-490, 2009.

18. Huo N, Ichikawa Y, Kamiyama M, Ishikawa T, Hamaguchi Y, Hasegawa S, Nagashima Y, Miyazaki K and Shimada H: MMP-7 (matrilysin) accelerated growth of human umbilical vein endothelial cells. Cancer Lett 177: 95-100, 2002.

19. Nishizuka I, Ichikawa Y, Ishikawa T, Kamiyama M, Hasegawa S, Momiyama N, Miyazaki K and Shimada H: Matrilysin stimulates DNA synthesis of cultured vascular endothelial cells and induces angiogenesis in vivo. Cancer Lett 173: 175-182, 2001.

20. Lee S, Jilani SM, Nikolova GV, Carpizo D and Iruela-Arispe ML: Processing of VEGF-A by matrix metalloproteinases regulates bioavailability and vascular patterning in tumors. J Cell Biol 169: 681-691, 2005.

21. Clasper S, Royston D, Baban D, Cao Y, Ewers S, Butz S, Vestweber D and Jackson DG: A novel gene expression profile in lymphatics associated with tumor growth and nodal metastasis. Cancer Res 68: 7293-7303, 2008.
22. Yoshitomi H, Kobayashi S, Ohtsuka M, Kimura F, Shimizu H, Yoshidome $\mathrm{H}$ and Miyazaki M: Specific expression of endoglin (CD105) in endothelial cells of intratumoral blood and lymphatic vessels in pancreatic cancer. Pancreas 37: 275-281, 2008.

23. Shiozaki K, Harada N, Greco WR, Haba A, Uneda S, Tsai H and Seon BK: Antiangiogenic chimeric anti-endoglin (CD105) antibody: pharmacokinetics and immunogenicity in nonhuman primates and effects of doxorubicin. Cancer Immunol Immunother 55: 140-150, 2006.

24. Tsujie M, Tsujie T, Toi H, Uneda S, Shiozaki K, Tsai H and Seon BK: Anti-tumor activity of an anti-endoglin monoclonal antibody is enhanced in immunocompetent mice. Int $\mathrm{J}$ Cancer 122: 2266-2273, 2008

25. Fonsatti E, Nicolay HJ, Altomonte M, Covre A and Maio M: Targeting cancer vasculature via endoglin/CD105: a novel antibody-based diagnostic and therapeutic strategy in solid tumours. Cardiovasc Res 86: 12-19, 2010. 